\title{
Posterior Triangle Lymph Node Group (Sublevels VA and VB)
}

National Cancer Institute

\section{Source}

National Cancer Institute. Posterior Triangle Lymph Node Group (Sublevels VA and VB).

NCI Thesaurus. Code C132513.

This group is composed predominantly of the lymph nodes located along the lower half of the spinal accessory nerve and the transverse cervical artery. The supraclavicular nodes also are included in the posterior triang le group. The superior boundary is the apex formed by the convergence of the sternocleidomastoid and trapezius muscles; the inferior boundary is the clavicle; the anterior (medial) boundary is the posterior border of the sternocleidomastoid muscle; and the posterior (lateral) boundary is the anterior border of the trapezius muscle. Thus, sublevel VA includes the spinal accessory nodes, whereas sublevel VB includes the nodes following the transverse cervical vessels and the supraclavicular nodes, with the exception of the Virchow node, which is located in level IV. The posterior triangle nodes are at greatest risk for harboring metastases from cancers arising from the nasopharynx, oropharynx, and cutaneous structures of the posterior scalp and neck. (AJCC 8th ed.) 